\title{
A Direct-Elution Technique for the Recovery of Thin-Layer Samples
}

\begin{abstract}
An elution technique is described which permits the recovery of samples from flexible thin-layer chromatograms without removal of the layer from the plate. This simple method avoids many of the problems associated with component removal and achieves high recovery with extremely small solvent volumes.
\end{abstract}

The separation of compounds by chromatography is often followed by the need to separate the compounds from the stationary phase for quantification, rechromatography, or other analytical procedures. In thin-layer chromatography compounds are usually separated from the adsorbent by removal of the layer from the backing by scraping or suction, followed by elution of the compounds in columns or by centrifugation (1-4). These procedures are generally quite time-consuming, and care is needed to prevent cross-contamination or loss of sample by air currents. Kirchner (5) stated that these problems could be alleviated if the compounds were eluted directly off the plate. Recently, Falk and Krummen (6) designed such a direct-elution apparatus, which is now marketed by Camag. They point out the following advantages of their instrument over the usual elution methods: (i) Time and money are saved; (ii) only small volumes of solvent are required; (iii) no centrifugation or filtration step is needed; (iv) elution is automatic once the apparatus is set up; (v) adsorbent remains unchanged in the eluted area.

In this report we describe a simple, direct-elution technique for flexible, thin-layer plates, which is based on the conventional methods for elution from paper chromatograms by descending solvent flow $(7,8)$. This device can be made from inexpensive and easily obtained materials and has the same advantages that were listed above for the more complex instrument.

\section{METHODS}

Description of device. The apparatus for the elution of thin-layer samples is described in Fig. 1. It consists of a transparent elution chamber, modified plastic syringes to hold the eluting solvent, rubber sealers which are cut to hold the thin-layer sections, and capillary tubes to collect the eluate. 




FIG. 1. Apparatus for direct elution of thin-layer samples. The elution chamber consists of a transparent, hard plastic box with a tight-fitting, removable top. Plastic syringes $(10 \mathrm{cc})$ are mounted in the top of the box along a line $4 \mathrm{~cm}$ from the main wide wall. The holes in the top should be a size that will hold the syringes at varying levels by frictional force. (The holes can be made by using a heated cork borer to melt through the plastic. If the hole becomes slightly too large, a hot dissecting needle can be used to draw out a small portion of plastic for a point of frictional contact.) A glass rod extends between both end walls, and on the main wide wall, capillary tubes are inserted through small holes that correspond horizontally with the placement of each syringe. The needle end of the syringe barrel is cut off to make an open tube of equal diameter throughout. The rubber sealer of the plunger is removed, and a straight slit about $5 \mathrm{~mm}$ long is carefully made in the rubber tip with a sharp razor blade. The slit should penetrate through the rubber into the space formerly occupied by the end of the plunger.

Analytical procedure. Samples are run on flexible, thin-layer plates, and the separated compounds are cut out in rectangular sections. Each rubber sealer is squeezed laterally to open the slit into which one corner of the thin-layer section is then inserted. The syringe barrels are placed in the openings in the top of the box, and the sealers containing the thin-layer sections are placed in the inner ends of the barrels. Solvent is poured in the bottom of the chamber to saturate the atmosphere, and the top is placed on the box. Narrow capillary tubes (e.g., 50 or $100 \mu \mathrm{l}$, Microcaps, Drummond Scicntific Co.) are inserted through the small holes and under the glass rod. Because of the thickness of the glass rod, the capillaries have a very slight downward slope inward, 
ensuring that the liquid to be collected in the capillary tubes will remain in contact with the thin-layer sections. The syringe barrels are positioned so that each capillary tube touches the bottom tip of a thinlayer section. One or two drops of a slurry consisting of 1 part silica gel $\mathrm{G}$ to 4 parts elution solvent is placed in the well of one rubber sealer into which projects the corner of the thin-layer section. Solvent is added promptly into the syringe barrel with care not to disturb the silica gel plug. This procedure is repeated for each syringe. The amount of silica gel may require a slight adjustment depending on the type of layer and the solvent used, since solvent channelling occurs with insufficient silica gel, and a slow flow rate occurs with an excess of silica gel.

\section{RESULTS}

Glucose samples were run on Eastman silica gel sheets and on Gelman ITLC sheets. Each sample contained $40 \mu \mathrm{g}$ of C-14 glucose with an activity of $14,000 \mathrm{dpm}$. The chromatograms were run in the upper phase of 1-butanol-acetic acid-water (4:1:5, by volume). By autoradiography the sugar regions were revealed on the Eastman layer at an $R_{f}$ value of 0.31 and on the Gelman layer at an $R_{f}$ value of 0.56 . The samples were cut from the layers in rectangular sections, which were then eluted with water as described above. Four regions on the Eastman layer and three on the Gelman layer were analyzed under the above conditions and counted in a Beckman 100C liquid scintillation counter. Both types of layers gave similar elution patterns and high recovery in small volumes. On the average $85.1 \%( \pm 3.3 \% \mathrm{SD})$ was eluted in the first $10 \mu \mathrm{l}$, and $96.6 \%( \pm 1.3 \% \mathrm{SD})$ was eluted in the first $40 \mu \mathrm{l}$. The $40-\mu \mathrm{l}$ elution took between 1 and $2 \mathrm{hr}$ to complete. The nonrecovered portion was determined by scraping off the layer after elution and counting its activity separately.

The effectiveness of this system was checked with a nonaqueous solvent by using methanol to observe visually the elution of dye samples on thin-layer sections. With the Gelman layer the flow characteristics and the color elution were similar to the previous results. With the Eastman layer, however, the flow rate was unacceptably slow, which resulted from the evaporation of the methanol on the layer. This problem was easily corrected by placing a portion of the plastic backing (layer removed) on each section so that the silica gel was sandwiched between the two laycrs of plastic. The backing was cut in such a way that the silica gel remained uncovered on the part of the section extending into the rubber sealer and on the bottom tip where the eluate was collected. The plastic pieces were held in place by paper clips. This sandwich technique would appear to have applicability in speeding up the elution rates with a wide range of volatile solvents. 


\section{DISCUSSION}

The technique presented here incorporates the general advantages described earlier of direct-elution systems. A special advantage of this technique is the unusually small, solvent volumes that are needed to get high recovery. This feature is particularly useful if the samples are to be rechromatographed, especially since the samples can be spotted directly from the capillary tubes. Another advantage is that the technique places little restriction on spot size or spot separation. In the system of Falk and Krummen (6), the elutable spot areas are limited to those that can be circumscribed on the plate by the instrument's elution cylinders. In the proposed technique any distinguishable spot area may be cut out and eluted.

The main limitation of this system is that it cannot be applied to all layers. Since the layer and backing must be quite thin in order to seal properly in the syringe and must be able to be cut into sections, this technique is unusable for glass plates. In addition we found that a particular flexible thin-layer, Eastman cellulose, gave unsatisfactory results, since the elution flow would often slow down and eventually stop. This may have been due to the rubber sealer squeezing through the soft cellulose layer. Working within the defined limitations, however, we found this technique to be a very convenient and effective method for the quantitative removal of samples from thin-layer chromatograms.

\section{REFERENCES}

1. Bobbitt, J. M., Schwarting, A. E., and Gritter, R. J. (1968) Introduction to Chromatography, pp. 81-83, Reinhold, New York.

2. Court, W. E. (1968) in Quantitative Paper and Thin-Layer Chromatography (Shellard, E. J., ed.), pp. 29 -49, Academic Press, New York.

3. Gänshirt, H. (1965) in Thin-Layer Chromatography (Stahl, E., ed.), pp. 44-57, Springer-Verlag, New York.

4. Sudilovsky, O., and Hinderaker, P. H. (1972) Anal. Biochem. 45, 525-529.

5. Kirchner, J. G. (1975) J. Chromatogr. Sci. 13, 558-563.

6. Falk, H., and Krummen, K. (1975) J. Chromatogr. 103, 279-288.

7. Vaněček, J. (1961) in Laboratory Handbook of Chromatographic Methods (Mikěs, O., ed.), pp. 63-65, Van Nostrand, London.

8. Sherma, J., and Zweig, G. (1971) Paper Chromatography and Electrophoresis, Vol. 2, pp. 93-95, Academic Press, New York.

Paul A. Adams

KeVIN B. KURTZ 